\section{The Temporo-Mandibular Joint}

Edited by Bernard G. Sarnat. Pp. 260. 2nd Edition. Springfield, Illinois: Charles C. Thomas. 1964. $\$ 12.50$.

Thirteen years ago the first edition of this work was published and the present edition bears witness to the considerable increase of knowledge which has accumulated during this period. Dr. Sarnat remains the editor and eight other specialists have contributed to the present text.

To those who have interested themselves in this important subject the present work will be warmly welcomed as a comprehensive study both from the academic and practical aspects. This book is very fully illustrated and these illustrations and diagrams help out the text. The references are especially full and such references are cited in the text, which is not always the case. It is very satisfactory to note the inclusion of an assessment of the evolution embryology and the comparative anatomy of this joint by Dr. E. Lloyd Du Bruhal: it is from these basic studies and from the functional adaptations of the masticatory system that a wider understanding of interpretation and clinical diagnosis can be possible. The study of pathology, histopathology, radiology are illuminating and stimulating. Perhaps the paramount value of all these studies to the specialist, orthopædic surgeon, orthodontist and dental practitioner are the chapters on diagnosis and treatment techniques, both surgical and dental. Neurophysical considerations are mentioned somewhat scantily but indeed this is too controversial a subject for inclusion in a work of this nature. It is rather unfortunate that British workers in this field recieve but little attention. The contributions of George Hankey are noted but Terence Ward's work upon the surgical treatment is not. Electromyographic studies make no mention of the very valuable and significant contributions by Wyke and Greenfield on disorders of masticatory refiexes and the anatomical and neurohistological examination of the innervation of the temporo-mandibular joints: an extensive body of research carried out in the Department of Physiology of the Royal College of Surgeons of England. Finally, this present work will remain a standard for some time and is a very valuable addition to our knowledge of an intricate study.

\section{Surgery of the Biliary Tract, Pancreas $\&$ Spleen}

Charles B. Puestow. Third edition. Pp. vii + 389, illustrated. London: Lloyd-Luke Medical Books. 1964. 75s.

This is the third edition of the book originally published in 1953, and the author in the preface states there have been extensive revisions in the sections on pancreatic surgery and on biliary tracto surgery. There are, however, several criticisms of the. book which should have been ironed out in previous $\Rightarrow$ ? editions. Throughout the volume the physiology and? particularly the anatomy of the organs is given ino very vague 'Reader's Digest' form. There is too much등 repetition of unnecessary material, measurements are $\overline{\bar{c}}$ given in either centimetres or inches indiscriminately, drug dosages in grains or milligrams. In $\mathbb{\perp}$ particular, the advances in hepatic surgery haven been due to the increased knowledge of the anatomye of the biliary tract and vascular structures of the $\overrightarrow{0}$ liver, and both angiography and cholangiography playa big part in modern surgery of the liver. These are $\vec{\omega}$ barely mentioned in the volume. Another drawbacko in a specialised volume is the complete lack of statistics to support or reject the procedures? described. There are some odd personal ideas put.

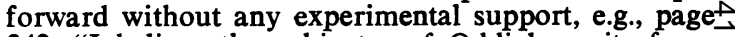
242 , "I believe the sphincter of Oddi loses its function after an adequately performed cholecystectomy. The operation must be sufficiently extensive to inter-c rupt the nerve fibres to the sphincter of Oddi".

The volume is a useful review of the subject of biliary surgery but it is rather fuller than is requiredo by students and certainly not extensive enough to be an operative manual for established surgeons. ${ }_{c}$ The section on the treatment of pancreatitis ing particular is very useful but would be so much morevaluable if authentic figures of results were available

\section{Introduction to Obstetrics}

George Herbert Green. 2nd edition. Pp. 205 45 illustrations. Christchurch, New Zealand N. M. Peryer. 1963. 32s. 6d.

The book is designed to give a student nursê an understanding of the basic principles of midwifery while she is undergoing her obstetric training. The text however is so full and it is so wels written that it will also serve pupil midwives and medical students who are learning the subject, of revising it prior to their examinations. There is little. change in the text of the new edition, the first nines chapters being devoted to anatomy, physiology and normal pregnancy and labour, while eight chaptersidescribe the common abnormalities that occur 3 Two chapters are devoted to the infant, but the care of the premature baby has been omitted. The diagrams are clearly drawn and well labelled, whilst the production of the book and the quality of theg paper are first class. 